\section{Cancer mortality in Latin America: implications for prevention}

\author{
Cristina Bosetti1 \\ and Carlo La Vecchia²
}

Two papers in this issue of the Revista Panamericana de Salud Pública/Pan American Journal of Public Health address the problem of detection and screening strategies for cancer prevention. One paper is on the diffusion of Pap smear tests for cervical cancer prevention in a high-risk area of South America (1), and the other is on prostate-specific antigen (PSA) and digital rectal examinations in the diagnosis of prostate cancer (2). A third study in this issue analyzes the incidence of cancer among Hispanic children in the United States of America, including mainly neoplasms of the lymphoid and hematopoietic system, many of which are amenable to treatment (3). These screening and diagnostic tests, as well as modern treatments for leukemias and Hodgkin's lymphomas, are largely available in developed countries, such as those of North America (Canada and the United States) and Western Europe. As illustrated by the paper by Dzuba et al. (1), there have been some delays in the adoption of organized programs and strategies for cancer prevention and treatment in some countries of Central America and South America. Even in the United States there are racial and ethnic disparities in cancer incidence and mortality $(3,4)$, which suggests that not all segments of the population in the country benefit equally from progress in the prevention, early detection, and treatment of cancer.

A recent paper provided an overview summary of cancer mortality in the Americas over the 1970-2000 period, with relevant implications for further investigation and public health intervention (5). Age-standardized (world population) mortality rates, derived from the World Health Organization (WHO) database (6), were presented for several cancers in 10 countries of Latin America with available and updated mortality and population data-Argentina, Brazil, Chile, Colombia, Costa Rica, Cuba, Ecuador, Mexico, Puerto Rico (United States), and Venezuela-plus in Canada and the United States, for comparative purposes.

This comprehensive analysis showed widely diverging patterns in total cancer mortality in the countries of Latin America, which reflect the variable mortality and trends for site-specific cancers. In 2000 the highest total male cancer mortality was seen in Argentina and Chile, with rates comparable to those of North America, i.e., about 155/100 000. For women, Chile and Cuba had the highest rates in Latin America (both over 100/100 000), again comparable to those of North America. These rates reflect the high mortality from cancer of the stomach (for Chile), lung and prostate (for Cuba) in men, stomach and cervix uteri (for Chile), and intestines and lung (for Cuba) in women. Colombia, Ecuador, and Mexico had the lowest male cancer mortality rates, due to low mortality from stomach, colorectal, and lung cancer. For women, the lowest rates were in Brazil and Puerto Rico, reflecting their low stomach and cervical cancer rates. In Argentina, Chile, Colombia, Costa Rica, and Venezuela, cancer mortality rates tended to decrease between 1970 and 2000, particularly among men. Rates were stable in Ecuador and Puerto Rico, and they increased in Cuba and Mexico.

With reference to head and neck cancers, in the year 2000 the highest rates for oral/pharyngeal cancer mortality in men were in Brazil, Cuba, and Puerto Rico, with values around 5-6/100 000, i.e., 1.5-2.0-fold higher than the values from North America. Colombia, Ecuador, and Mexico showed the lowest rates, with values around 1-2/100 000. As in North America and several countries of Europe (7), a leveling out, or even a fall, in mortality from oral/pharyngeal cancer occurred in various countries of Latin America between 1990 and 2000. Mortality rates for cancer of the mouth/pharynx in women were very low (0.5$1.5 / 100$ 000) in most countries, and showed no appreciable change between 1970 
and 2000. Recent declines in mortality from cancer of the oral cavity/pharynx in Latin America $(8,9)$ are likely due to decreased exposure to the two major risk factors: alcohol drinking and tobacco smoking. Additional factors, mainly related to improvements in diet, may have also played some role in the recent trends.

With reference to esophageal cancer, in 2000, Argentina, Brazil, Chile, and Puerto Rico had the highest male mortality rates (about 7-8/100 000). The same countries also had high esophageal mortality rates in women (2-3/100 000). Ecuador and Mexico showed very low mortality rates from esophageal cancer in both men and women. In most Latin American countries, rates steadily declined in both sexes over the 1970-2000 period. In Cuba and Mexico, as well as in North America, increases in rates were seen in men, but not in women. Further, mortality patterns were similar for esophageal and mouth/pharyngeal cancers, underlining the similarities in risk factors for these two neoplasms, including alcohol drinking, tobacco smoking, and dietary habits $(8,9)$.

In recent years, male stomach cancer mortality was exceedingly high in most Latin American countries (rates over 10/100 000). While Argentina, Cuba, Mexico, and Puerto Rico had male rates lower than 10/100 000, those rates were still higher than the 3.7/100 000 value in the United States. Stomach cancer mortality in women was lower than in men, but the geographical pattern was similar. Thus, mortality rates from stomach cancer were higher in Chile, Costa Rica, and Ecuador (over 12/100 000), and lower in Argentina, Cuba, and Puerto Rico (below 4/100 000). The corresponding figures in North America were about 23/100 000. Mortality from stomach cancer steadily decreased over the 1970-2000 period in most countries of Latin America. The reasons for this favorable trend in gastric cancer rates are not completely understood, but they may include better food conservation and refrigeration, a more varied and affluent diet, reduced prevalence of Helicobacter pylori infection, and (at least in men) a possible reduction in tobacco smoking (9). However, mortality from stomach cancer is still extremely high in several Latin American countries, underlining the importance of continuous monitoring and of efforts to prevent and manage this malignancy.

Mortality rates from cancer of the intestines in 2000 were below 10/100 000 in most countries of Latin America, with the exceptions of Argentina, Cuba, and Puerto Rico. Corresponding rates in North America were around 14$15 / 100000$ in men, and around 10/100 000 in women. However, while mortality from colorectal cancer in North America declined steadily between 1970 and 2000, the trend in the countries of Latin America was less favorable. The low rates for colorectal cancer in countries such as Brazil, Colombia, and Mexico, if not due to substantial underreporting, likely reflect long-term improvements in dietary habits in these countries. However, trends in colorectal cancer mortality are unfavorable in most countries of Latin America, probably because of modifications in dietary and lifestyle habits, including the increased prevalence of overweight and obesity. The relatively high colorectal cancer mortality in Argentina is consistent with the role of red meat (frequently consumed in this country) in colorectal carcinogenesis. Unexpectedly, in Cuba, as in Costa Rica and Ecuador, colorectal cancer mortality was higher in women than in men. It appears that Cuban women not only have higher rates of tobacco- and alcohol-related neoplasms, but also of colorectal cancer, which is moderately associated with alcohol and tobacco consumption, but is mainly related to nutrition.

In 2000, lung cancer mortality rates in men were much lower in most Latin American countries (below 21/100 000) than in North America (over 40/100 000). The highest rates for men in Latin America were registered in Argentina and Cuba (over 35/100 000), while the lowest one was in Ecuador (7.7/100 000). In Argentina, rates steadily, though moderately, decreased between 1970 and 2000. In most other Latin American countries, as in North America, mortality from lung cancer has shown a tendency to level off since the 1980s. Only in Ecuador and Venezuela is mortality from lung cancer still increasing. 
With the exception of Cuba, in 2000, female lung cancer rates were still below 10/100 000 in most Latin American countries, that is, substantially lower than in North America (over 20/100 000). However, in several countries of Latin America, as in the United States, lung cancer mortality in women increased between 1970 and 2000. Since the lung is the most common tobacco-related cancer site (9), these trends essentially reflect the smoking habits of men and women in the Latin American countries. The comparatively low rates of lung cancer mortality among women in most Latin American countries indicate the importance of the tobacco control interventions that have been undertaken.

In 2000, female breast cancer mortality was highest in Argentina, with rates higher than those reported in North America (around 18/100 000). In all other Latin American countries, rates were around 12-15/100 000, with the exception of Colombia, Ecuador, and Mexico, where rates were below 10/100 000. Over the 1970-2000 period, breast cancer mortality rates were stable in Argentina, Chile, and Cuba, but increased in other Latin American countries, particularly those that used to have lower rates. In North America over the same period, breast cancer mortality rates steadily declined, particularly among middle-aged women. The rise in female breast cancer rates in some of the Latin American countries is likely related to changes in Latin American women's reproductive habits, particularly decreased fertility. However, changes in dietary habits and other lifestyle factors, such as increased overweight, may also have played some role. Further, advanced therapies should be more widely adopted in the countries of Latin America. These therapies include tamoxifen and adjuvant chemotherapy, as well mammography screening for early diagnosis. The therapies and the screening have contributed to the leveling off and subsequent decrease in the mortality from breast cancer in North America and Europe $(4,7)$.

Uterine cancer mortality was exceedingly high in Latin America in 2000, essentially due to high rates of cervical cancer. Rates were over 15/100 000 (over 30/100 000 in women aged 35-64 years) in Ecuador, Mexico, and Venezuela, and over 10/100 000 (over 20/100 000 for ages 35-64) in most other countries. Uterine cancer mortality rates in North America were around 4/100 000 (7/100 000 for ages 35-64). With the exception of Argentina and Cuba, there was a steady fall in uterine cancer mortality in most Latin American countries, particularly among middle-aged women, beginning in the 1970s. These declines were likely due to a decrease in cervical cancer mortality, following some adoption (though still largely inadequate) of Pap smear screening in these countries. The excess mortality in most Latin America nations indicates the need for more adequate screening programs, which would limit the consequences of this avoidable cause of death.

In 2000, Cuba and Venezuela had the highest prostate cancer mortality rates (over 20/100 000), followed by Chile and Costa Rica, with rates around $20 / 100$ 000. The lowest mortality rates were in Brazil and in Mexico, with levels comparable to those of North America, that is, around 12-13/100 000 for all ages, and 5-6/100 000 for those 35-64 years old. Between 1970 and 2000, overall prostate cancer mortality showed a moderate increase in all the countries of Latin America. However, no noticeable changes in rates were seen in men below age 65 . Therefore, it is likely that the overall trends are influenced by recent changes in diagnosis and certification of prostate cancer, following the introduction of PSA testing. This is particularly true for Chile, Costa Rica, Ecuador, and Venezuela, where rates at all ages and rates for those 35-64 years old substantially diverged. In order to reduce prostate cancer mortality in Latin America, it is important that diagnostic advances and modern therapeutic approaches be adopted. These should include transurethral resection of the prostate, as well as hormonal therapy and radiotherapy for patients with locally advanced prostate cancer.

In 2000, testicular cancer mortality rates in Latin America ranged from 0.3/100 000 in Cuba to 1.3/100 000 in Chile. In North America, as in Europe (7), mortality rates steadily declined between 1970 and 2000, when they were about 
$0.2 / 100000$. Testicular cancer mortality is now appreciably higher in Latin America than in North America. This reflects an inadequate adoption of modern, platinum-based chemotherapy regimens (10), which have substantially reduced mortality from testicular cancer in developed areas of the world since the early 1970s. Again, this calls for urgent adoption of modern treatment for a largely avoidable cause of death among young men in Latin America.

Male mortality rates from leukemias were between 3 and 5/100 000 in 2000 in the countries of Latin America, that is, slightly lower than the rate of 56/100 000 in North America. Values in Latin American women were between 2 and $4 / 100000$, that is, comparable to those in North America. In North America there was a steady decrease in the rates beginning in the early 1970s, following the adoption of integrated therapy regimens, mainly for children and young adults (4). The trends in the countries of Latin America were less consistent, with a decline in Argentina, but increases in Ecuador and Mexico. Again, this would suggest some delay in the adoption of effective modern therapy schemes in the Latin American countries, although it is possible that trends in mortality rates from leukemias were also influenced by improved accuracy of diagnosis and certification.

In conclusion, the comprehensive analysis of cancer mortality in Latin American (5) showed that mortality from some common cancers (including colorectal and lung) is still low in Latin America as compared to North America. In addition, in several Latin American countries, declines in rates have been seen for some cancers (including of the stomach, uterus, and lung, plus other tobaccorelated cancers) over the 1970-2000 period. However, mortality rates from female lung and breast cancers have been increasing in most countries of Latin America, and several of these countries showed greatly elevated mortality from cancer of the cervix. Reducing cancer mortality in Latin America in the near future would therefore require an integrated strategy of prevention focused on control of tobacco and alcohol, particularly for Cuba and Brazil; adoption of a richer and more favorable diet so as to prevent stomach cancer, especially in Chile, Colombia, and Costa Rica; and more widespread early cervical cancer screening in most Latin American countries. Another priority for most countries of Latin America is the adoption of therapeutic advances for selected neoplasms that are amenable to treatment, including testicular cancer and leukemias.

\section{REFERENCES}

1. Dzuba IG, Calderón R, Bliesner S, Luciani S, Amado F, Jacob M. A participatory assessment to identify strategies for improved cervical cancer prevention and treatment in Bolivia. Rev Panam Salud Publica. 2005;18(1):53-63.

2. Berger VW, Semanick L. Refining the assessment of the sensitivity and specificity of diagnostic tests, with applications to prostate cancer screening and non-small cell lung cancer staging. Rev Panam Salud Publica. 2005;18(1):64-70.

3. Wilkinson JD, Gonzalez A, Wohler-Torres B, Fleming LE, MacKinnon J, Trapido E, et al. Cancer incidence among Hispanic children in the United States. Rev Panam Salud Publica. 2005;18(1):5-13.

4. Jemal A, Clegg LX, Ward E, Ries LA, Wu X, Jamison PM, et al. Annual report to the nation on the status of cancer, 1975-2001, with a special feature regarding survival. Cancer. 2004;101:3-27.

5. Bosetti C, Malvezzi M, Chatenoud L, Negri E, Levi F, La Vecchia C. Trends in cancer mortality in the Americas, 1970-2000. Ann Oncol. 2005;16:489-511.

6. World Health Organization Statistical Information System. WHO mortality database. Available from: http:/ / www3.who.int/whosis/menu.cfm [Web site]. Accessed 22 June 2005.

7. Levi F, Lucchini F, Negri E, Boyle P, La Vecchia C. Cancer mortality in Europe, 1995-1999, and an overview of trends since 1960. Int J Cancer. 2004;110:155-69.

8. Corrao G, Bagnardi V, Zambon A, La Vecchia C. A meta-analysis of alcohol consumption and the risk of 15 diseases. Prev Med. 2004;38:613-9.

9. International Agency for Research on Cancer. Tobacco smoke and involuntary smoking. Lyon: IARC; 2004. (IARC Monographs on the Evaluation of Carcinogenic Risks to Humans and their Supplements, Vol. 83).

10. Levi F, Lucchini F, Boyle P, Negri E, La Vecchia C. Testicular cancer mortality in Eastern Europe [letter]. Int J Cancer. 2003;103:574. 